\title{
SIMULATION STUDY OF INTERCONNECTION NETWORKS WITH VIRTUAL CUT-THROUGH ROUTING
}

\author{
Yelena Rykalova \\ Department of Computer Science \\ UMass Lowell \\ 220 Pawtucket St, \\ Lowell, MA, USA \\ yelena_rykalova@uml.edu
}

\author{
Lev B. Levitin
}

ECE Department
Boston University,
8 St.Mary's St,
Boston, MA, USA
levitin@bu.edu

\begin{abstract}
A simple network model with torus topology and the virtual cut-through routing has been considered in order to find out and analyze certain relationships between network parameters, load and performance. Simulation experiments for various values of network parameters (mesh size, message path length, and message length) have been performed. It is found that if the mesh linear dimension is at least twice as large as the message path length (the distance from source to destination) the network behavior (latency and saturation point) does not depend on the mesh size. Both theoretical and empirical results show that the saturation point is inversely proportional to the message length. If the network is in the steady state, a good agreement with Little's theorem has been observed.
\end{abstract}

Keywords: computer interconnection networks, network torus topology, virtual cut-through routing, latency, saturation in networks.

\section{INTRODUCTION}

Many commercially available parallel computers use extensive communications between otherwise independent nodes. This communications network is implemented as a network of interconnected routers (each having its local processor) (Kleinrock 1975; Kiasari, Lu, and Jantsch 2013; Chen et al. 2011; Minkenberg 2013). Various routing techniques are used in interconnection networks (Kaushal and Singh 2014; Duato et al. 2001; Wang et al. 2014). Store-and-forward approach is based on the assumption that an entire message must be received at any intermediate node before it can be forwarded to the next node. Obviously, for a long message, the total delivery time may turn out to be quite large. To the contrary, in wormhole routing, each message is divided into small packets - flits. The header flit contains information about source and destination, and is routed through the network according to this information and routing algorithm. Other flits of the message follow the header flit. When header flit of the message is blocked at an intermediate node because the requested link is occupied by another message, the flits are buffered at each node along the path up to the current node. This forms a long "worm" which remains in the network blocking other messages, thereby increasing their delivery time. Also, the problem of deadlocks emerges in this approach and should be dealt with (Levitin, Karpovsky, and Mustafa 2009; Karpovsky, Levitin, and Mustafa 2014).

Virtual cut-through (VCT) routing algorithm, first introduced by Kermani and Kleinrock (1979), is supposed to mitigate the drawbacks of both the above-mentioned techniques. Unlike the wormhole approach, in VCT routing, if the next node cannot accept the message, the current node must still be able 
to buffer the rest of the incoming message from previous nodes. Thus the VCT algorithm achieves a much higher throughput and avoids deadlocks at the expense of increased buffer capacity.

Several papers were devoted to the comparison between different routing techniques (Kaushal and Singh 2014). Analytical models of interconnection networks were considered in (Kiasari, Lu, and Jantsch 2013; Nikitin and Cortadella 2009; Rexford and Shin 1996; Kodgire and Shiurkar 2015). Certain practical implementations were described in (Chen et al. 2011, Sadawarte, Gaikwad, and Patrikar 2011; Hag et al. 2015). Network latency in VCT networks is defined as the average time from the moment a message is generated by the source processor to the moment when the last flit of the message enters the consumption channel of the destination processor. The network latency consists of propagation delay, router delay, and contention (blockage) delay.

In this paper, we study network latency and saturation using the VCT routing policy. At each network node, we introduce (unlimited) storage buffers. The "unlimited" buffer model means in practice that the network throughput is limited by the link occupancy (utilization), rather than by the buffer capacity. So, after the header is blocked, the "worm" collapses ("condenses") into the storage buffer. This method prevents deadlocks and improves network performance (increases bandwidth, decreases latency, delays saturation).

\section{COMMUNICATION NETWORK MODEL}

The specific features of our model (different from others published) are described in this section. The following assumptions have been made for the network model implementation:

- The storage buffers are unlimited and use FIFO (first-in, first-out) policy.

- The same clock is used for all network nodes.

- When message is generated, it takes one time unit for its header (if not blocked) to appear at the router internal input port.

- It takes two time units (a time unit can include one or more clock cycles) to move a header flit from a router input port to its output port.

- It takes one time unit to move a flit (except the header) from a router input port to its output port.

- It takes one time unit to move a flit from the router output port to the input port of the next node (to go through the link).

\subsection{Network topology model}

In many systems where the VCT routing may be used, the physical distance between communication nodes is small and thus unimportant. In such systems, real network topology can be abstracted without loss of generality as easily constructed lower-dimension meshes and tori. This paper deals with two-dimensional torus networks. The symmetry of toroidal networks leads to a more balanced utilization of communication links than "open" mesh topologies and improves scalability. Each node in such a network consists of a router and a local processor. Each router has four external input/output (I/O) channels, and one internal input/output channel to the local processor. All I/O channels are bidirectional so that two messages may travel simultaneously in the opposite directions between the nodes.

\subsection{Routing}

Each input/output port of the router contains three buffers: input buffer, output buffer and output storage buffer. All input and output buffers can hold only one flit at a time. In our model, the storage buffer (which is an extension of the output buffer) is assumed to be "unlimited". This means that it can hold as many messages as needed at each moment of time. The local processor has the same one-flit sized input and output buffers. Suppose that the local processor generates message $M_{1}$ at time $t_{1}$, and another message $M_{2}$ at time $t_{2}\left(t_{2}>t_{1}\right)$. It is possible that at time $t_{2}$ the router is still processing message $M_{1}$ and link from the local processor to the router is busy. To handle this situation, we introduce an unlimited storage buffer like 
the one at the external port, so the local processor can store the message $M_{2}$ in its output storage buffer until the link is free. As a result, external and internal ports have the same three-buffer architecture. This enabled us to use the same software implementation for external and internal ports. It is believed that in real-life implementation the router processor needs more time (more time units) to route header flit (to decode destination, look up routing table, and decide on which output port to send) then to route remaining flits. We model this asymmetry by assigning two time units in the router for routing the header flit. Because all other flits of the message just follow the header, it takes one time unit in the router to send them to the correct output port.

Network contention occurs if two messages compete for the same channel. VCT networks generally outperform store-and-forward networks when the traffic patterns are sparse, but suffer substantial contention (leading to performance degradation) when the traffic is heavy. One way to address the issue of contention is to use adaptive routing, which allows a message to take an alternative path when primary path (defined in the routing table) is blocked by another message.

We use the deadlock-free adaptive unicast VCT routing algorithm as described below.

- Every router has a (static) two-dimensional routing table relating minimum length path from each of the router's four output ports to each network node.

- The routing table is used to perform dynamic routing based on the following set of rules:

- If the current node (node to which the message header has arrived) is the destination, the header is routed to the internal port connecting to the local processor.

- Else header of the message is sent from the input port to the output port which has the shortest path to the destination node.

- In general, more than one output port may have the minimum distance to destination, so the header is routed to the first available (free) port, where "first" refers to the port with the smallest number.

- If all ports with minimum distance to the destination are busy, the router sends the header to the storage buffer of that one from these ports whose number is the largest one.

- If more than one simultaneously arrived headers should be routed to the same output port based on the rules described above, the header of the message with the smallest identification number will be processed first, and the header(s) of message(s) with larger identification number(s) will remain in the output storage buffer.

- Flits follow the header. If header motion is blocked, the header is routed to the storage buffer, all flits follow the header and accumulate (condense) in the storage buffer.

This routing algorithm assures that every link in the message path is occupied no longer than time equal to the message length.

\subsection{Message generation}

We assume that at each time unit, every node in the network can generate a message with probability $\lambda$ (the message generation rate) independently of all other nodes. Destination nodes for generated messages are selected randomly among nodes having the specified distance $l$ from the source node. Obviously, increasing $\lambda$ increases the network load (the number of messages simultaneously traveling in the network), which, in turn, leads to the latency growth until network saturation is reached.

\section{THEORETICAL BACKGROUND}

\subsection{The network states}

We consider three different network states: startup, steady state, and saturation. 
When network simulation starts up, initially there are no messages in the system. Then, new messages start appearing in the network. Even in the absence of other messages, certain time $\tau_{\min }$ is required for a message to reach its destination. During this time more messages can be generated, so initially after the startup the number of messages in the network increases. When network reaches its steady state, the average number of messages generated during time $\Delta t$ equals the average number of messages delivered during the same time $\Delta t$, so that the number of messages in the system (in transit from source to destination) becomes approximately constant over time. For a meaningful evaluation of the latency, network must reach its steady state before data on the network behavior should be collected.

The network load increases with the number of messages present in the network and with the message length, since longer messages occupy links for longer time. As a result, for each message length, the network can accommodate only a limited range of message generation rates. If message generation rate is too high, the number of messages generated during time $\Delta t$ exceeds the number of messages that can be delivered during this time, and the number of messages in the system is increasing with time with no bound. Thus, the latency tends to infinity, and network becomes dysfunctional. This is the state of saturation.

The theoretical analysis of the network performance with the VCT routing is a challenging problem. In (Levitin and Rykalova 2018), an expression for the saturation point (message generation rate at which network saturates) and approximate expressions for the latency (under the assumptions of the "mean field theory" have been obtained.

The critical value $\lambda_{c r}$ is given by

$$
\lambda_{c r}=\frac{4}{l m} .
$$

Here $m$ is the length of the message (in flits) and $l$ is the distance (number of hops) from the source to the destination. (It is taken into account that the number of links in a bidirectional toroidal network with $n$ nodes is $4 n$.)

The approximate expression for the latency $\tau$ is

$$
\tau=(l+1)\left(\frac{\lambda l m}{4-\lambda l m}+3\right)+m .
$$

Expression (2) shows that transition to saturation is a second-order (continuous) phase transition with a critical exponent equal to 1, in agreement with the "mean field" theory.

\section{SIMULATION}

\subsection{Simulation procedure}

The relatively small network sizes have been chosen in order to reduce the simulation time. The distances from source to destination and the message length varied for different experiments, but were assigned prior to the simulation and kept constant during simulation run.

The network performance is characterized by latency (average delivery time) as a function of the network load, and by its saturation load, which describes maximum network capacity. The latency is obtained by averaging delivery times for all messages generated during time $T$. The time $T$ is selected according to the value of $\lambda$ so that the total number of messages generated in the system during time $T$ per each destination node will be about the same for all values of $\lambda$ (in our experiments this number is about 120). Since the average number of messages generated per unit of time is proportional to $\lambda$ the following empirical formula has been used to estimate $T: T \approx 10 \times 4 l / \lambda$.

To ensure that the network is in its steady state during data collection period, the delivery time was recorded for messages generated within time interval $\left(t_{\min }, t_{\min }+T\right)$, where $t_{\min }$ was sufficiently large. 


\section{Rykalova and Levitin}

\subsection{Simulation results}

In each simulation run, we analyzed the number of messages in the network as a function of time to determine network state and make sure that is computed using data collected in the steady state.

Message latency was analyzed as a function of network load (as parameterized by $\lambda$ ) using message lengths of 5, 10 and 20 flits for mesh sizes ranging from $n=4 \times 4$ to $n=12 \times 12$ (network linear size 4, 6, 8, 12). To ensure steady state, $t_{\min }=50000$ was used.

The observed latency values are close for meshes of different sizes. For all mesh sizes considered, network is still in the steady state for $\lambda=0.14$, but close to the saturation. For $\lambda=0.15$, steady state could not be reached at $t_{\min }=50000$ : number of messages in the system increased approximately linearly during the interval $\left(0, t_{\min }\right)$. It suggests that saturation is reached between $\lambda=0.14$ and $\lambda=0.15$.

The network latency for $l=3$ (3-hop path) and $m=5$ is shown in Figure 1. Again, similar behavior is observed for all mesh sizes for small values of $\lambda$; however, for the smallest $(n=4 \times 4)$ mesh latency quickly increases with $\lambda$ and saturation is reached at some point between $\lambda=0.12$ and $\lambda=0.13$. The atypical behavior observed in the smallest $4 \times 4$ mesh, seemingly, is due to the fact that the path distance exceeds $1 / 2$ of the linear dimension of the network. These results indicate that network behavior (latency and saturation point) does not depend on the mesh size if the mesh linear dimension $s$ is at least twice the message path length $s \geq 2 l$.

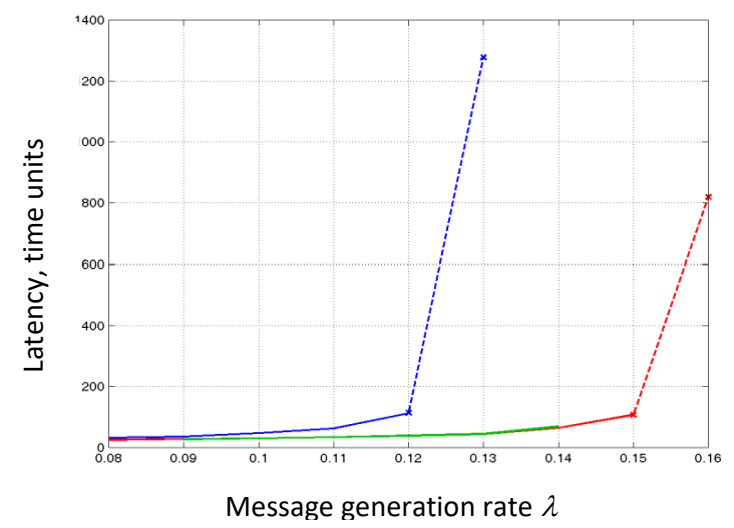

Figure 1: Latency for message length $m=5$ flits. Path length $l=3$ hops. Solid line: steady state; dashed line: steady state could not be reached. Mesh size $4 \times 4$ (blue line), $6 \times 6$ (red line), $8 \times 8$ (green line).

Next, simulations were performed using $l=2$ and $l=3$ with longer messages. Results for $l=2$ and message length of $m=10$ and $m=20$ flits are shown in Figures 2 and 3, respectively. Since $s \geq 2 l$ for $s=6,8,12$ and $l=2$, we expect similar network behavior, while the results for $d=2$ are atypical $(s<2 l)$ and demonstrate early saturation.

Simulation results for $l=3$ are shown in Figures 4 and 5 for message lengths $m=10$ and 20, respectively. Again, similar network behavior is observed, when $s \geq 2 l$ condition is satisfied. These results indicate that network behavior (latency and saturation point) does not depend on the mesh size if the mesh linear dimension $s$ is at least twice the message path length $s \geq 2 l$. Results for latency dependence on the network load look somewhat paradoxical. It is seen that, if $\lambda$ is small, latency is larger for longer paths. However, when $m=5$, latency for the shorter path becomes larger for large values of $\lambda$, and saturation occurs earlier for the shorter path. Possibly, this behavior is related to the fact that the message length $(m=5)$ is commensurate with the distance from the source to destination. Seemingly, this effect disappears if the length of the message is large enough $(m=20)$. 


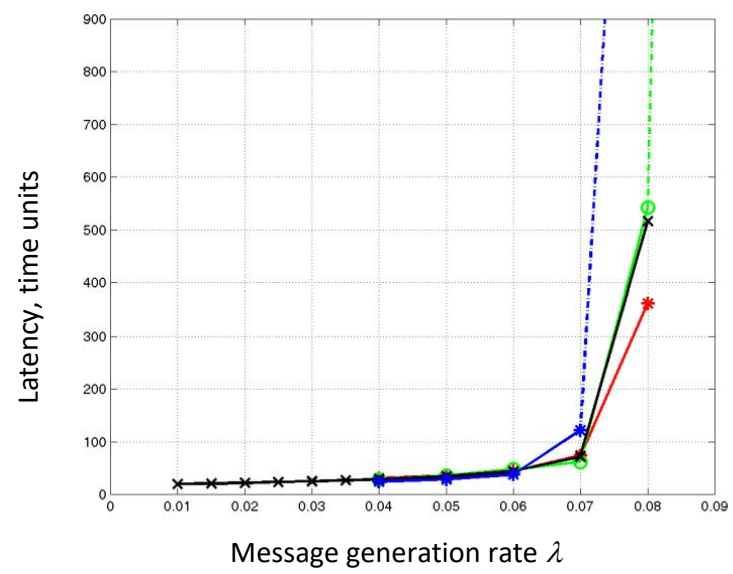

Figure 2: Latency for message length $m=10$ flits. Path length $l=2$ hops. Solid line: steady state; dashed line: steady state could not be reached. Mesh size $2 \times 2$ (blue line), $4 \times 4$ (red line), $6 \times 6$ (green line), and $8 \times 8$ (black line).

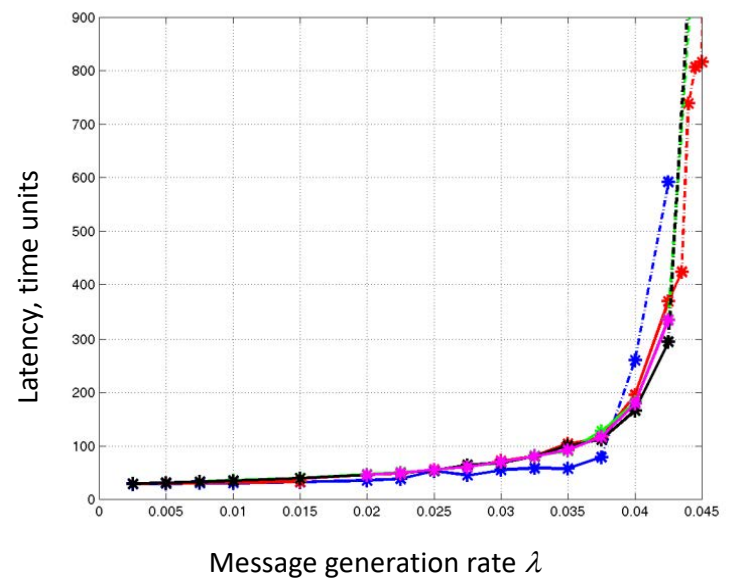

Figure 3: Latency for message length $m=20$ flits. Path length $l=2$ hops. Solid line: steady state; dashed line: steady state could not be reached. Mesh size $2 \times 2$ (blue line), $4 \times 4$ (red line), $6 \times 6$ (green line), $8 \times 8$ (black line), and $12 \times 12$ (magenta line).

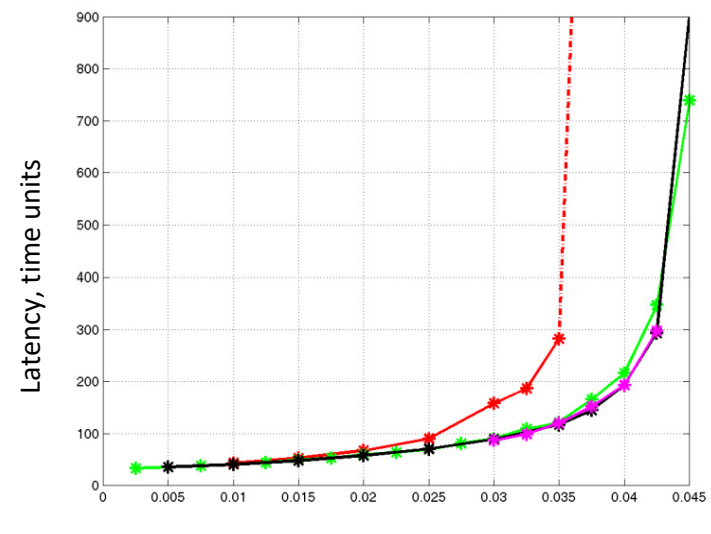

Message generation rate $\lambda$ 
Figure 4: Latency for message length $m=10$ flits. Path length $l=3$ hops. Solid line: steady state; dashed line: steady state could not be reached. Mesh size $4 \times 4$ (red line), $6 \times 6$ (green line), $8 \times 8$ (black line).

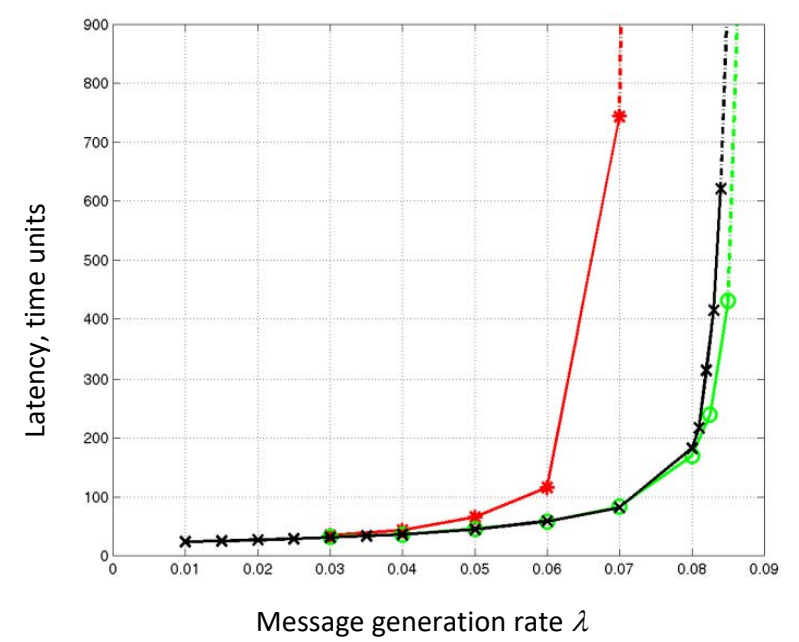

Figure 5: Latency for message length $m=20$ flits. Path length $l=3$ hops. Solid line: steady state; dashed line: steady state could not be reached. Mesh size $2 \times 2$ (blue line), $4 \times 4$ (red line), $6 \times 6$ (green line), $8 \times 8$ (black line), and $12 \times 12$ (magenta line).

Network latencies obtained for $l=2$ and $l=3$ are compared in Figure 6 and Figure 7. Results on latency dependence of the network load look somewhat paradoxical. It is seen that, if $\lambda$ is small, latency is larger for longer paths. However, when $m=5$, latency for the shorter path becomes larger for larger values of $\lambda$, and saturation occurs earlier for the shorter path. Possibly, this behavior is related to the fact that the message length $(m=5)$ is commensurate with the distance from the source to destination. Seemingly, this effect disappears if the length of the message is large enough $(m=20)$.

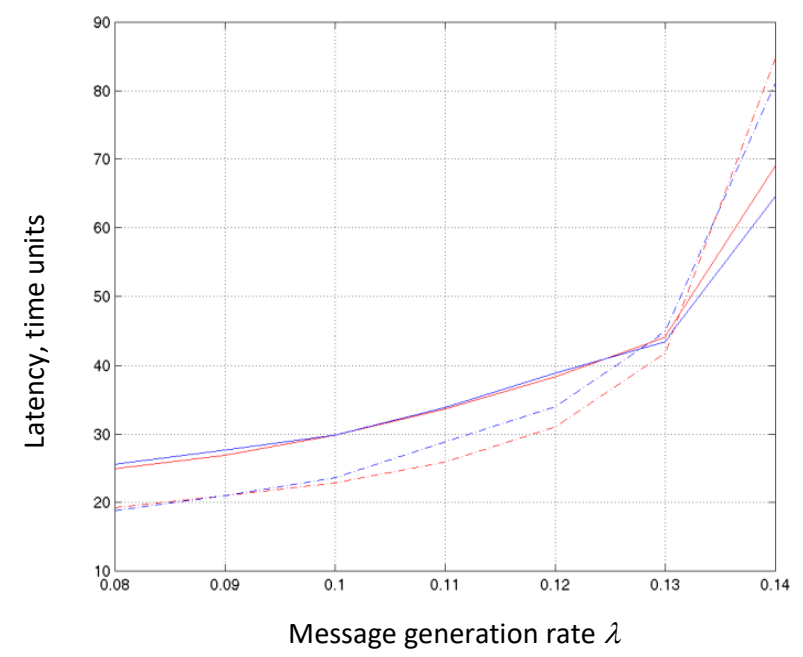

Figure 6: Latency for message length $m=5$ flits. Mesh sizes $6 \times 6$ (blue lines) and $8 \times 8$ (red lines). Solid lines: $l=3$, dashed lines: $l=2$. 


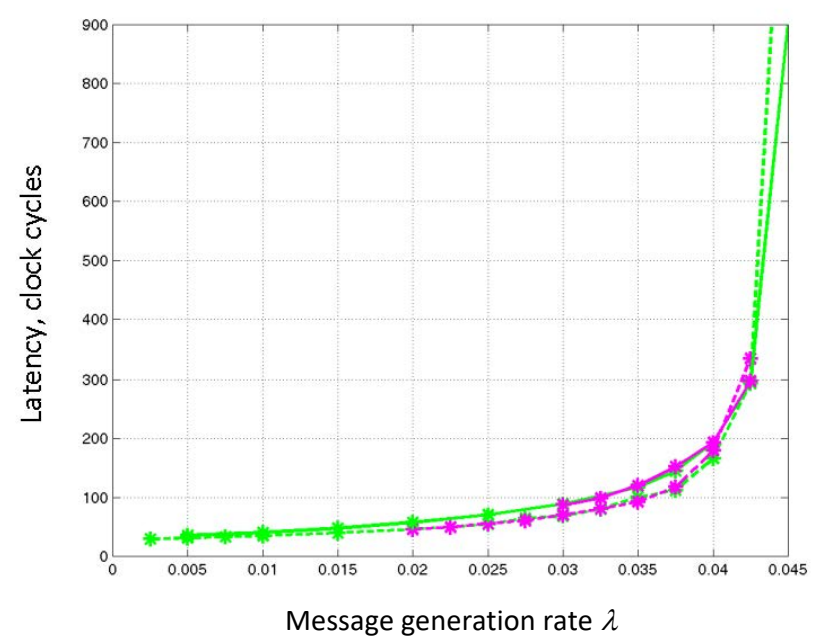

Figure 7: Latency for message length $m=20$. Mesh sizes $8 \times 8$ (green lines) and $12 \times 12$ (magenta lines). Solid line: $l=3$, dashed line: $l=2$.

\subsection{Dependence of the message latency and saturation on the message length}

For the same message generation rate $\lambda$, longer messages result in higher network loads. Thus, we expect larger latencies and earlier saturation for longer messages.

Message latencies for the 2-hop path in $4 \times 4,6 \times 6$ and $8 \times 8$ meshes are shown in Figure 8 for message lengths $m=5$ (blue), $m=10$ (red), and $m=20$ (green). Results are shown using star symbols $\left({ }^{*}\right)$ for $4 \times 4$ mesh, circles ( $(\circ)$ for $6 \times 6$ mesh, and crosses $(\times)$ for $8 \times 8$ mesh.

Similar results for 3 hops paths are shown in Figure 9. Obviously, the network load increases with the message length. Let's hypothesize that the network load is proportional to the product of the message length $m$ and the message generation rate $\lambda$. Then one can expect that the message generation rate at which network saturates $\left(\lambda_{c r}\right)$ is inversely proportional to the message length $m$. The theoretical foundation for the hypothesis is given in Section 3.

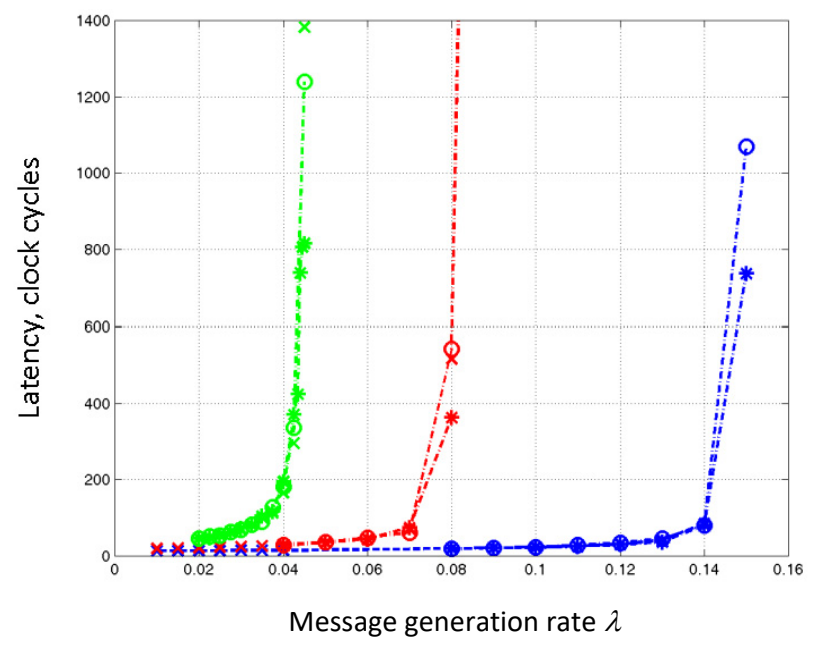

Figure 8: Latency as function of $\lambda$ for $l=2$ and various message lengths: $m=5$ (blue lines), $m=10$ (red lines), $m=20$ (green lines). Mesh sizes: $4 \times 4\left(^{*}\right), 6 \times 6(\circ), 8 \times 8(\times)$. 


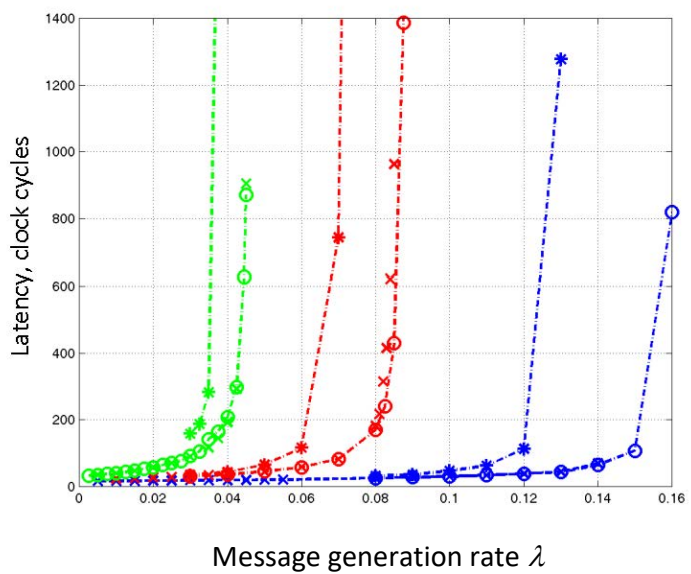

Figure 9: Latency as function of $\lambda$ for $l=3$ and various message lengths: $m=5$ (blue lines), $m=10$ (red lines), $m=20$ (green lines). Mesh sizes: $4 \times 4\left(^{*}\right), 6 \times 6(0), 8 \times 8(\times)$.

The results of our numerical experiments are in a good agreement with this hypothesis (see Figure 10). It is seen that for all cases, when $s \geq 2 l$ the dependence of $\lambda_{c r}$ of the message length $m$ can be closely approximated as $\lambda_{c r}=0.8 / m$ (solid red line in Figure 10).

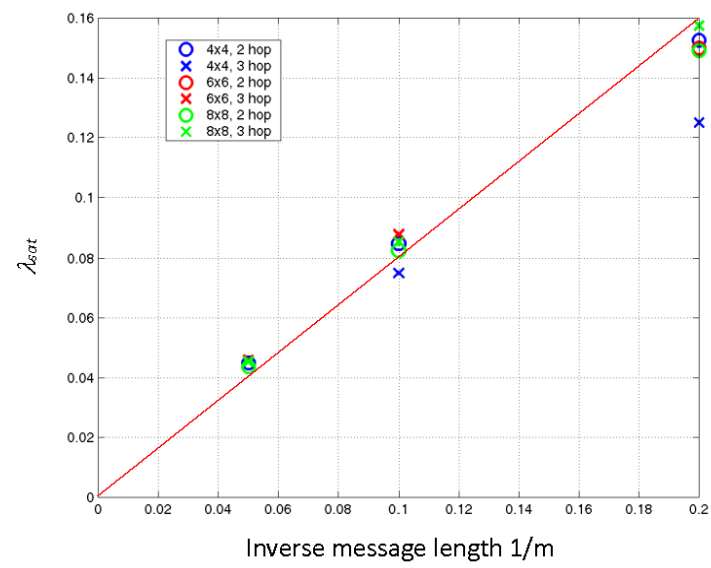

Figure 10: Message generation probability at which network saturates $\left(\lambda_{c r}\right)$ as a function of the inverse message length $1 / m$. Red line $\lambda_{\text {sat }}=0.8 / \mathrm{m}$ shown for reference.

\subsection{Number of messages in the system as function of message load}

In the steady state, the relationship between the average number of messages in the system and the latency is given by Little's theorem (Kleinrick 1975), $N=\lambda n \tau$.

Here $N$ and $\tau$ are expected values of two random variables: number of messages in the network $N_{s}$, sampled over the total period of observation, and the sample delivery time $\tau_{s}$. Therefore, the values of $N_{s}$ and $\tau_{s}$ fluctuate with time and the relationship between $N_{s}$ and $\tau_{s}$ satisfies Little's theorem only approximately.

We have measured values of $N_{s}$ by averaging the number of messages in the network from $t_{\text {min }}$ up to the end of simulation, as well as calculated the number of messages in the network using the observed values of $\tau_{s}$. As shown in Figures 11 and 12, the directly measured values of $N_{s}$ and those calculated by the use of Little's theorem are in a very good agreement, which supports the validity 
of the simulation experiments. However, note that when network state is closed to saturation, the calculated number of messages usually exceeds the measured value.

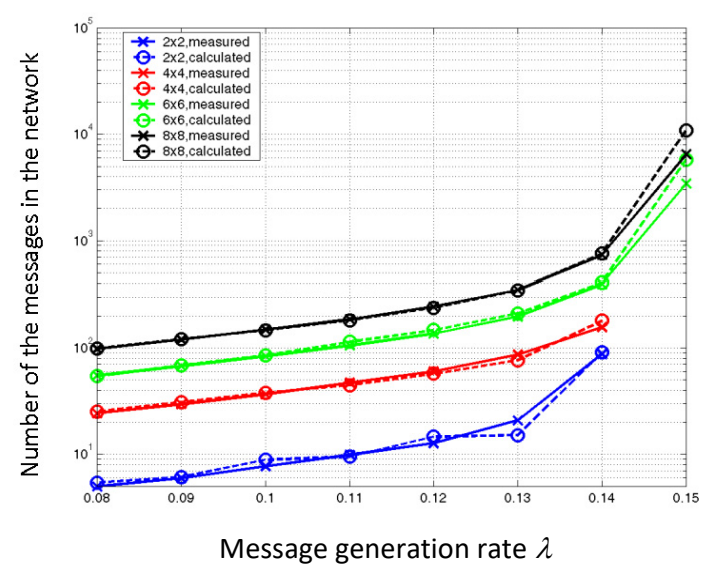

Figure 11: The number of the messages in the network as function of $\lambda$. Solid line: measured during simulation; dashed line: calculated by the use of Little's theorem. Message length $m=5$ flits. Path length $l$ $=2$ hops.

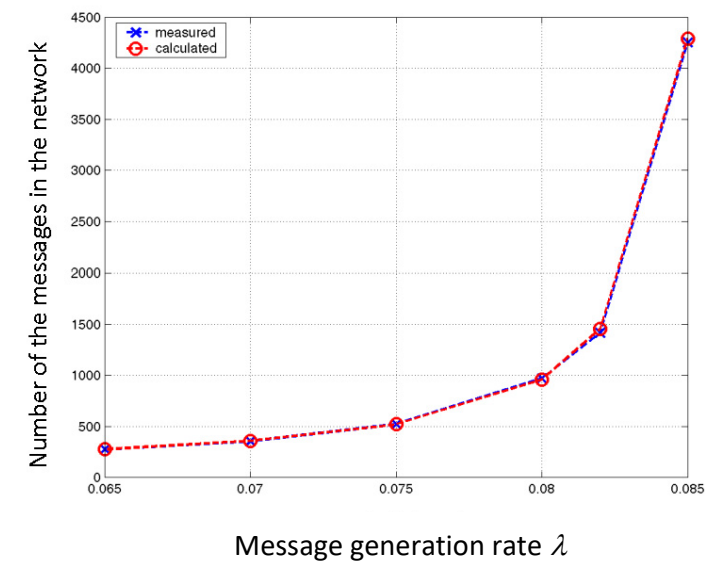

Fig. 12. The number of messages in the network as function of $\lambda$. Mesh size $8 \times 8$. Message length $m=10$ flits. Path length $l=3$ hops.

\section{CONCLUSION}

A model of a 2-dimensional toroidal interconnection network with virtual cut-through routing has been studied. Analytical expression for the saturation point and approximate expressions for the network latency for the ranges of small network loads and loads close to the critical value have been obtained.

- The critical value of the probability of message generation $\lambda=\lambda_{c r}$ is inversely proportional to the distance between the source and the destination $l$ and the length of messages $m$ : $\lambda_{c r}=\frac{4}{l m}$.

- The latency $\tau$ at the saturation point experiences a second-order (continues) phase transition with the critical exponent equal to 1 .

- For small values of $\lambda$, the latency grows as a linear function of $\lambda$. 
Simulation experiments have been performed in order to find out and analyze certain empirical relationships that can be used as a starting point for a deeper theoretical analysis and further research. In particular, the following results have been obtained.

- Network behavior (latency and saturation point) does not depend on the mesh size if the mesh is "large enough" compared to the path length. As an appropriate criterion, the mesh linear dimension should be at least twice as large as the message path length: $s \geq 2 l$.

- For the same message generation rate, latency increases and saturation occurs earlier for longer messages. It appears that the saturation point $\lambda_{c r}$ (message generation rate at which network saturates) is inversely proportional to the message length. If the condition $s \geq 2 l$ is satisfied, numerical results are in a good agreement with a simple empirical relation $\lambda_{c r}=0.8 / \mathrm{m}$ independently of the mesh size. (It seems to be consistent also with the theoretical expression (1)).

- If the network is in the steady state, the independently measured number of messages $N_{s}$ and the average delivery time $\tau_{s}$ are in a good agreement with Little's theorem for their expected values $N$ $=\lambda n \tau$.

\section{REFERENCES}

Amandeep Kaushal and Sarbdeep Singh. 2014. "Network on Chip Architecture and Routing Techniques: A survey". International Journal of Research in Engineering and Science (IJRES) ISSN (Online): 2320-9364, ISSN (Print): 2320-9356 www.ijres.org Volume 2 Issue 6, June 2014, pp.65-79.

Amandeep Kaushal, Sarbdeep Singh. 2014. "Network on Chip Architecture and Routing Techniques: A survey". In International Journal of Research in Engineering and Science (IJRES) ISSN (Online): 2320-9364, ISSN (Print): 2320-9356 www.ijres.org Volume 2 Issue 6, June 2014, pp.65-79.

Chen, D., et al. 2011. "The IBM Blue Gene/Q interconnection network and message unit," International Conference for High Performance Computing, Networking, Storage and Analysis (SC), Seatle, WA, 2011, pp. 1-10.

Duato, J., Robles, A., Silla, F., \& Beivide, R. 2001. "A Comparison of Router Architectures for Virtual Cut-Through and Wormhole Switching in a NOW Environment". J. Parallel Distrib. Comput. 61, 2 (February 2001), 224-253.

Duato, J., Robles, A., Silla, F., and Beivide, R. 2001. "A Comparison of Router Architectures for Virtual Cut-Through and Wormhole Switching in a NOW Environment". J. Parallel Distrib. Comput. 61, 2 (February 2001), pp. 224-253. DOI=http://dx.doi.org/10.1006/jpdc.2000.1679

Hag, A. A. Y., Hafizur Rahman, M.M., Nor, R. M., Sembok, T. M. T., Miura, Y., and Inoguchi, Y. 2015. "Uniform Traffic Patterns using Virtual Cut-Through Flow Control on VMMN". In Procedia Computer Science, Volume 59, 2015, pp. 400-409.

Karpovsky, M. G., Levitin, L. B., and Mustafa, M. 2014. "Optimal Turn Prohibition for Deadlock Prevention in Networks with Regular Topologies". In IEEE Trans. On Control of Network Systems, v. 1, 1, 2014, pp. 74-85.

Kermani, P., Kleinrock, L. 1979. "Virtual Cut-Through: A New Computer Communication Switching Technique". Computer Networks, Volume 3, Issue 2, (Sept. 1979), pp. 267-286.

Kiasari, A. E., Lu, Z., and Jantsch, A. 2013. "An analytical latency model for networks-on chip". IEEE Trans. Very Large Scale (VLSI) Syst., 21(1), pp. 113-123.

Kiasari, A. E., Lu, Z., and Jantsch, A. 2013. "An analytical latency model for networks-on chip". IEEE Trans. Very Large Scale (VLSI) Syst., 21(1), pp. 113-123.

Kleinrock, L. 1975. Queueing Systems Volume I: Theory. New York: Wiley. 
Kodgire, S., and Shiurkar, U. 2015. "An Analytical Router Model for Networks-On-Chip". In IOSR Journal of VLSI and Signal Processing (IOSR-JVSP) Volume 5, Issue 4, Ver. II (Jul - Aug. 2015), 1621 e-ISSN: 2319 - 4200, p-ISSN No. : 2319 - 4197.

Levitin, L. B., Karpovsky, M. G., and Mustafa, M. 2009. "Minimal Sets of turns for Breaking Cycles in Graphs Modeling Networks". In IEEE Trans. Parallel and Distributed Systems, v. 21, 9, 2010, pp.13421353.

Levitin, L.B. and Rykalova, Y. 2018. "Analysis and simulation of networks with virtual cut-through routing". In Proceedings of the 2018 IAJC International Conference, ISBN 978-1-60643-379-9. http://cd16.iajc.org/

Levitin, L.B., \& Rykalova,Y. "Latency and phase transitions in interconnection networks with unlimited buffers”. International Journal of Modern Engineering (IJME), Vol. 17, No.1, 2016, 70-77.

Minkenberg, C. 2013. Interconnection Network Architectures for High-Performance Computing. Advanced Computer Networks - Guest Lecture, 21 May 2013, IBM. (C) 2013 IBM Corporation.

Nikitin, N., and Cortadella, J. 2009. "A performance analytical model for Network-on-Chip with constant service time routers". In Proceedings of the 2009 International Conference on Computer-Aided Design (ICCAD '09). ACM, New York, NY, USA.

Rexford, J., \& Shin, K.G. 1996. Analytical Modeling of Routing Algorithms in Virtual Cut-Through Networks. U Michigan.

Rykalova, Y., \& Levitin, L. 2017. "Phase Transitions in Interconnection Networks with Finite Buffers". International Journal of Modern Engineering (IJME), Vol. 17, No.2, 2017, 33-40.

Rykalova, Y., Levitin, L. B., and Brower, R. 2010. "Critical phenomena in discrete-time interconnection networks". Physica A: Statistical Mechanics and its Applications, vol. 389(22), pp. 5259-5278

Sadawarte, Y. A., Gaikwad, M. A., and Patrikar, R. M. 2011. "Implementation of Virtual Cut-Through Algorithm for Network on Chip Architecture". In IJCA Proceedings on International Symposium on Devices MEMS, Intelligent Systems \& Communication (ISDMISC) (1): pp. 5-8.

Wang, P., Ma, S., Lu, H., and Wang, Z. 2014. "A comprehensive comparison between virtual cut-through and wormhole routers for cache coherent Network on-Chips". In IEICE Electronics Express. 11. 20140496-20140496. 10.1587/elex.11.20140496.

Wang, P., Ma, S., Lu, H., and Wang, Z. 2014. "A comprehensive comparison between virtual cut-through and wormhole routers for cache coherent Network on-Chips". IEICE Electronics Express. 11. 20140496-20140496. 10.1587/elex.11.20140496.

\section{AUTHOR BIOGRAPHIES}

LEV B. LEVITIN is currently Distinguished Professor of Engineering Science with the Department of Electrical and Computer Engineering at Boston University, Boston, MA. He has published over 200 papers, presentations, and patents. His research areas include information theory; quantum communication systems; physics of computation; quantum computing; quantum theory of measurements, mathematical linguistics; theory of complex systems; coding theory; theory of computer hardware testing, reliable computer networks, and bioinformatics. He is a Life Fellow of IEEE, a member of the International Academy of Informatics and other professional societies. His email address is levitin@bu.edu.

YELENA RYKALOVA is currently Assistant Professor at UMass Lowell, MA and Visiting Researcher with the Department of Electrical and Computer Engineering at Boston University, Boston, MA. Her research interests are in computer networks, in particular, in application of concepts and models of statistical physics to the analysis of network performance. She is a member of IEEE and the Society for Modeling and Simulation International (SCS). Since 2008 she has been active in organization and preparation for the Spring Simulation Multiconference (SpringSim) as Technical Committee Member, Reviewer, and Publicity and Session Chair. Her email address is yelena_rykalova@uml.edu. 